\title{
Decompressive craniectomy in hemorrhagic cerebral venous thrombosis: clinicoradiological features and risk factors
}

\author{
*Si Zhang, MD, Hexiang Zhao, MD, Hao Li, MD, Chao You, PhD, and Xuhui Hui, PhD \\ Department of Neurosurgery, West China Hospital of Sichuan University, Chengdu, Sichuan, People's Republic of China
}

\begin{abstract}
OBJECTIVE Decompressive craniectomy (DC) is a life-saving treatment for severe hemorrhagic cerebral venous thrombosis (CVT). However, the correlations between the clinicoradiological features and surgical outcomes of this disease are not well established. Therefore, the authors endeavored to analyze the potential risk factors for this more severe subtype of CVT and to provide more evidence regarding the benefits of DC in patients with hemorrhagic CVT.
\end{abstract}

METHODS The clinical features, radiological findings, and surgical outcomes of patients with severe hemorrhagic CVT who had undergone DC treatment in the period from January 2005 to March 2015 were retrospectively analyzed, and the risk factors for this disease were evaluated.

RESULTS Fifty-eight patients, 39 females $(67.2 \%)$ and 19 males (32.8\%), with a mean age of $39.7 \pm 12.5$ years, were included in this study. The mean duration from symptom onset to surgery was $3.3 \pm 1.9$ days, and 21 patients experienced acute courses. On neuroimaging, the mean mass lesion volume was $114.7 \pm 17.7 \mathrm{ml}$. Nine patients had bilateral lesions, and 7 patients had deep CVT. According to their hemorrhagic proportion, cases were divided into hemorrhagedominated (27 [46.6\%]) and edema-dominated (31 [53.4\%]) groups. After 6 months of follow-up, 56.9\% of patients had achieved a favorable outcome, and 8 patients had died. The hemorrhage-dominated lesions $(p=0.026)$ and deep cerebral venous involvement $(p=0.026)$ were significantly associated with a poor outcome.

CONCLUSIONS In patients suffering from severe hemorrhagic CVT, DC is an effective life-saving treatment that is associated with favorable outcomes. Hemorrhage-dominated lesions and deep cerebral venous involvement have a significant impact on the outcome of this disease.

https://thejns.org/doi/abs/10.3171/2016.8.JNS161112

KEY WORDS cerebral venous thrombosis; decompressive surgery; intracerebral hemorrhage; vascular disorders

$\mathrm{C}$ EREBRAL venous thrombosis (CVT) is an uncommon cerebrovascular disorder accounting for < $1 \%$ of all strokes. ${ }^{6}, 18$ Young adults and children are more susceptible to this disease, especially individuals in the 3rd decade of life and neonates., ${ }^{2,5}$ Historically, atypical symptoms have often resulted in delayed diagnoses and ineffective treatment during the early stages of this disease. ${ }^{18}$ Recently, with the application of CT venography and MR venography, early recognition and treatment of this disease have become much more feasible, and anticoagulation therapy has led to encouraging reductions in mortality. ${ }^{6,18}$ However, it has been reported that $40 \%$ of patients with CVT can suffer from intracranial hypertension and that approximately 30\%-40\% can present with intracerebral hemorrhage. ${ }^{18}$ Refractory intracranial hypertension caused by hemorrhagic CVT is associated with rapid clinical deterioration and can lead to herniation. Therefore, aggressive interventions such as endovascular thrombolysis/thrombectomy and decompressive craniectomy (DC) have been introduced. ${ }^{16,21}$ Decompressive craniectomy, which is commonly performed in patients with malignant arterial stroke, can alleviate the refractory intracranial hypertension caused by severe hemorrhagic CVT and is thus considered a life-saving treatment in affected

ABBREVIATIONS CPP = cerebral perfusion pressure; $\mathrm{CVT}=$ cerebral venous thrombosis; $\mathrm{DC}=$ decompressive craniectomy; $\mathrm{GCS}=\mathrm{Glasgow}$ Coma Scale; $\mathrm{H} / \mathrm{L}=$ hemorrhage/lesion volume; $I C P=$ intracranial pressure; $\mathrm{mRS}=$ modified Rankin Scale.

SUBMITTED April 30, 2016. ACCEPTED August 1, 2016.

INCLUDE WHEN CITING Published online October 21, 2016; DOI: 10.3171/2016.8.JNS161112.

* Drs. Zhang and Zhao contributed equally to this work. 
patients. To date, however, no randomized trials regarding the benefits of DC in patients with CVT have been reported. Moreover, because of the small sample sizes and diverse inclusion criteria of previous studies, the indications for DC in patients with severe CVT are still not well established.

In the current study, we presented our 10-year institutional experience regarding DC for severe hemorrhagic CVT and evaluated the correlations between clinicoradiological features and surgical outcomes of this disease. The objectives of our study were to analyze the potential risk factors for this more severe subtype of CVT and to provide more evidence regarding the benefits of $\mathrm{DC}$ in patients with hemorrhagic CVT.

\section{Methods \\ Study Population}

The present study was approved by the West China Hospital Trials and Biomedical Ethics Committee.

The clinical records of patients who had undergone DC for severe hemorrhagic CVT in the period from January 2005 to March 2015 were retrospectively analyzed. Patients who met the following criteria were enrolled in this study: 1) those whose diagnosis of CVT was preoperatively confirmed on MR venography or CT venography, although digital subtraction angiography was selectively performed for patients with chronic clinical courses; 2) those with Glasgow Coma Scale (GCS) scores $\leq 9$; 3) those between 15 and 70 years of age; 4) those who underwent DC because of impending or established brain herniation, which may have presented as progressive neurological deterioration (GCS changes) associated with changes in pupil size and CT evidence of space-occupying lesions causing midline shifts or obliteration of the basal cisterns despite maximal medical therapy and the provision of intensive care; and 5) those who underwent DC with or without hematoma evacuation.

Exclusion criteria were as follows: 1) patients with bilateral mydriasis who had a critically endangered status; 2) patients with primary lesions in the cerebellum; and 3) patients with definitive surgical contraindications.

\section{Institutional Therapeutic Strategy}

Once the diagnosis of hemorrhagic CVT was confirmed, standard medical treatment was initiated, including anticoagulation treatment with subcutaneous low-molecular-weight heparin injected at a dose of 180 anti-factor Xa U/kg/24 hrs (anti-factor Xa causes fewer hemorrhagic complications and can be given at fixed doses without constant laboratory monitoring). Some patients who were referred from other hospitals had previously received intravenous unfractionated heparin; we continued their previous anticoagulation therapy. In the event of refractory intracranial hypertension warranting surgical intervention, anticoagulation treatment was temporarily interrupted, and protamine was administered to patients with prolonged coagulation times. Postoperative anticoagulation treatment was restarted or started 48 hours after surgery in the patients without evidence of worsening or new-onset hemorrhage on CT. Subsequent anticoagula- tion treatment was continued for 2 weeks, maintaining a doubled activated partial thromboplastin time.

Unilateral craniectomy was performed in our cohort, and evacuation of the hematoma was performed if it was associated with significant mass effect. Intraparenchymal ICP monitoring was recommended.

Postoperatively, stepwise intracranial pressure (ICP) management was performed in the neurological intensive care unit (maintaining ICP $<20 \mathrm{~mm} \mathrm{Hg}$ and $\mathrm{CPP}>60$ $\mathrm{mm} \mathrm{Hg}$ ). Conventional therapy included sedation, neuromuscular blockage, intubation, ventilation, $30^{\circ}$ head elevation, osmotic dehydration, and external ventricular drainage. Therapeutic hypothermia $\left(32^{\circ} \mathrm{C}\right.$ to $\left.34^{\circ} \mathrm{C}\right)$ was used in patients who suffered from refractory intracranial hypertension (ICP $>25 \mathrm{~mm} \mathrm{Hg}$ for more than 1 hour).

For convalescent patients, routine neurological rehabilitation and early cranioplasty at 2 months after surgery were performed in our institution.

\section{Data Collection and Statistics}

The following clinical features were documented: age, sex, predisposing factors, initial syndrome, duration from symptom onset to surgery, preoperative GCS score, pupillary response, and preoperative anticoagulation therapy. Neuroimaging experts evaluated preoperative radiological findings. The main imaging parameters included degree of midline shift, total volume of the lesion, presence of bilateral lesions, and involved veins. Total lesion volume, which was characterized by the volume of the hematoma and edematous brain tissue, was calculated using the $\mathrm{ABC} / 2$ method (ellipsoid equation) based on $\mathrm{CT}$ and MRI findings. Regarding the radiological features of hemorrhagic CVT, some patients would present with large areas of edema mixed with small, scattered, patchy hemorrhages, while others showed large areas of hemorrhage surrounded by moderate edema. To ensure proper measurement of the lesion, we characterized 2 lesion types describing the pathology associated with severe hemorrhagic CVT. Hemorrhage-dominated lesions were characterized by hemorrhage/lesion volume (H/L) ratios $>0.5$, while edema-dominated lesions were characterized by $\mathrm{H} / \mathrm{L}$ ratios $<0.5$ (Fig. 1).

The modified Rankin Scale (mRS) was applied to assess 6-month outcomes. For patients who experienced good neurological outcomes, the mRS assessment was completed in the outpatient department via face-to-face interview. For patients who could not attend the follow-up because of a poor neurological status, the assessment was performed via telephone interview with surrogates.

Univariate and multivariate regression analyses were performed to determine the significance of the relationships between preoperative variables and poor outcome (mRS Scores 3-6). The Student t-test was used to compare continuous variables, and Pearson's chi-square test was used to compare categorical variables. Multivariate regression was assessed in terms of odds ratios and $\mathrm{R}^{2}$ values. The Hosmer-Lemeshow method was used to check the calibration of the regression result. All statistical analyses were performed using SPSS software (version 19.0, IBM Corp.), and $\mathrm{p}<0.05$ was considered statistically significant. 

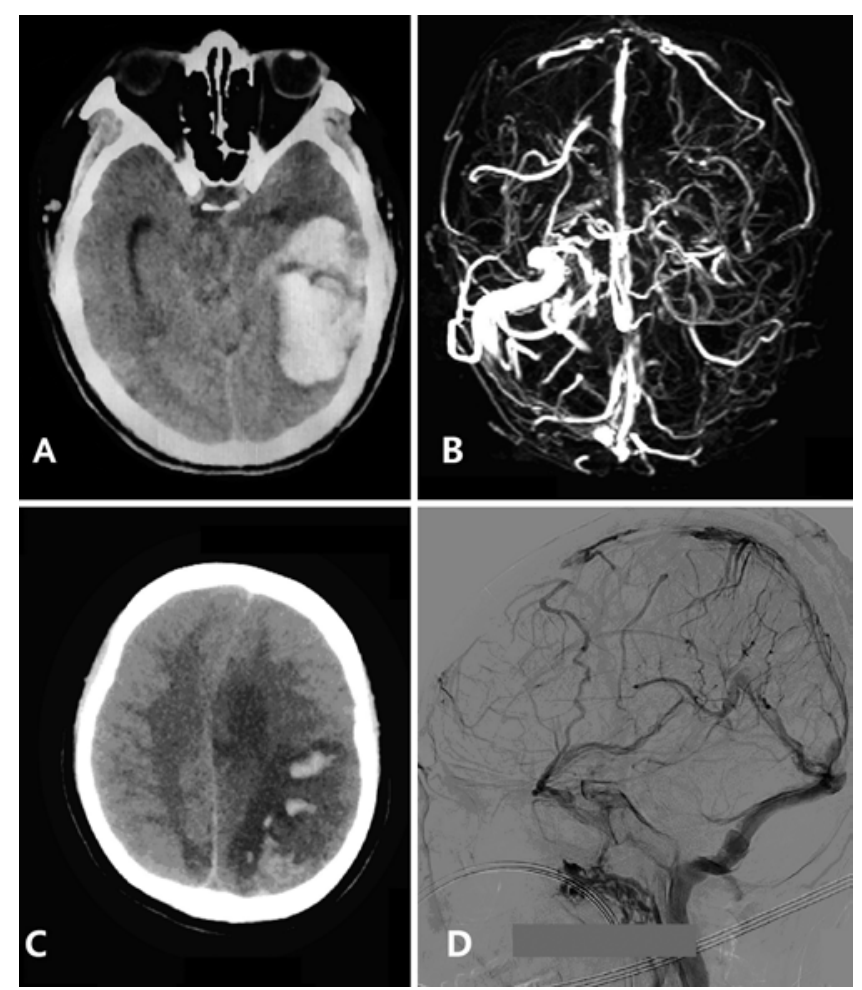

FIG. 1. Representative cases of the lesion types. A 34-year-old female suffered from sudden onset of seizure and impaired consciousness. Computed tomography showed a large hematoma located in the temporoccipital lobe with significant midline shift $(\mathbf{A})$. Magnetic resonance venography confirmed the diagnosis of transverse sinus thrombosis (B). This patient was classified as having a hemorrhagic-dominated lesion of CVT. A 23-year-old female complained of progressive headache for 2 weeks aggravated by impaired consciousness for 12 hours. Computed tomography showed a large area of edema mixed with small scattered patchy hemorrhage (C). Digital subtraction angiography revealed a superior sagittal sinus thrombosis (D). The patient was classified as having an edema-dominated lesion of CVT.

\section{Results}

\section{Clinical and Radiological Features}

From January 2005 to March 2015, a total of 422 patients with CVT were treated in the stroke unit in the West China Hospital of Sichuan University. Among these patients were 70 who had a surgical indication for DC and 201 patients $(47.6 \%)$ who presented with hemorrhagic lesions. After reviewing the patient medical data, we found 58 patients with severe hemorrhagic CVT who had undergone DC; these patients were eligible for our study. The clinical and radiological features of these patients are listed in Table 1. Overall, the 39 females $(67.2 \%)$ and 19 males (32.8\%) had a mean age of $39.7 \pm 12.5$ years (range 18-69 years). Notably, 8 patients were Tibetan, representing $13.8 \%$ of the patients in our series. Within 2 weeks of symptom onset, 12 patients (20.7\%) had predisposing factors, including oral contraceptive use in 8 , late puerperium in 3 , and breast abscess in 1 . The most common symptom was headache (39 patients [67.2\%]). Seventeen patients (29.3\%) suffered from seizures preoperatively, 13 of them from generalized seizures. Four patients (6.9\%)
TABLE 1. Clinicoradiological features of patients with severe hemorrhagic CVT

\begin{tabular}{|c|c|}
\hline Characteristic & Value \\
\hline Total no. of patients & 58 \\
\hline Mean age in yrs $( \pm S D)$ & $39.7 \pm 12.5$ \\
\hline Females (no. [\%]) & $39(67.2)$ \\
\hline Patients w/ predisposing factors (no. [\%]) & $12(20.7)$ \\
\hline \multicolumn{2}{|l|}{ Ethnicity (no. [\%]) } \\
\hline Han & $49(84.5)$ \\
\hline Tibetan & $8(13.8)$ \\
\hline Others & $1(1.7)$ \\
\hline \multicolumn{2}{|l|}{ Initial symptoms (no. [\%]) } \\
\hline Headache & $39(67.2)$ \\
\hline Seizure & $4(6.9)$ \\
\hline Disturbance of consciousness & $8(13.8)$ \\
\hline Focal neurological deficits & $7(12.1)$ \\
\hline Mean duration from onset to op in days $( \pm S D)$ & $3.3 \pm 1.9$ \\
\hline \multicolumn{2}{|l|}{ GCS score (no. [\%]) } \\
\hline 9 & $5(8.6)$ \\
\hline 8 & $12(20.7)$ \\
\hline 7 & $17(29.3)$ \\
\hline 6 & $15(25.9)$ \\
\hline 5 & $8(13.8)$ \\
\hline 4 & $1(1.7)$ \\
\hline Abnormal pupillary response (no. [\%]) & $47(81)$ \\
\hline Standard anticoagulation (no. [\%]) & $43(74.1)$ \\
\hline Midline shift $>5 \mathrm{~mm}(\mathrm{no} .[\%])$ & $43(74.1)$ \\
\hline Mean lesion vol in ml ( \pm SD) & $114.7 \pm 17.7$ \\
\hline Hemorrhage-dominated lesion (no. [\%]) & $27(46.6)$ \\
\hline Edema-dominated lesion (no. [\%]) & $31(53.4)$ \\
\hline Deep cerebral venous involvement (no. [\%]) & $7(12.1)$ \\
\hline \multicolumn{2}{|l|}{ Involved dural sinus (no. [\%]) } \\
\hline Sagittal sinus & $26(44.8)$ \\
\hline Transverse sinus & $12(20.7)$ \\
\hline Sigmoid sinus & $17(29.3)$ \\
\hline Multiple sinuses & $3(5.2)$ \\
\hline
\end{tabular}

reported seizure as their initial symptom, and 8 patients $(13.8 \%)$ listed disturbed consciousness as their initial symptom. Regarding the time from symptom onset to surgery (mean $3.3 \pm 1.9$ days), 21 patients (36.2\%) experienced acute deterioration within 48 hours of symptom onset, and 13 patients had prolonged preoperative courses lasting longer than 5 days (maximum 9 days). The mean preoperative GCS score was 6.79 , and abnormal pupillary responses were observed in 47 patients $(81 \%)$. Fifteen patients $(25.9 \%)$ received nonstandard anticoagulation in other hospitals.

Regarding radiological findings, all the patients presented with space-occupying mass lesions, and 9 presented with bilateral lesions. Midline shifts $>5 \mathrm{~mm}$ were observed in 43 patients $(74.1 \%)$. The mean lesion volume was $114.7 \pm 17.7 \mathrm{ml}$. Using $\mathrm{H} / \mathrm{L}$ ratios, we classified 27 patients 
TABLE 2. Univariate analysis of preoperative variables and clinical outcome

\begin{tabular}{|c|c|c|c|c|c|}
\hline Variable & Favorable Outcome & Poor Outcome & p Value & OR $(95 \% \mathrm{Cl})$ & $\mathrm{R}^{2}$ \\
\hline Mean age in yrs ( \pm SD) & $37.03 \pm 10.51$ & $43.20 \pm 14.25$ & 0.068 & $1.042(0.997-1.090)$ & 0.08 \\
\hline $\operatorname{Sex}($ no. $[\%])$ & & & 0.112 & & \\
\hline Male & $8(42.1)$ & $11(57.9)$ & & & \\
\hline Female & $25(64.1)$ & $14(35.9)$ & & & \\
\hline Predisposing factors (no. [\%]) & $6(50.0)$ & $6(50.0)$ & 0.588 & & \\
\hline Seizure (no. [\%]) & $10(58.8)$ & $7(41.2)$ & 0.849 & & \\
\hline Mean duration from onset to op in days ( \pm SD) & $4.03 \pm 2.02$ & $2.40 \pm 1.35$ & 0.003 & $0.555(0.374-0.823)$ & 0.249 \\
\hline GCS score (no. [\%]) & & & 0.724 & & \\
\hline $7-9$ & $20(58.8)$ & $14(41.2)$ & & & \\
\hline $4-6$ & $13(54.2)$ & $11(45.8)$ & & & \\
\hline Abnormal pupillary response (no. [\%]) & $25(53.2)$ & $22(46.8)$ & 0.239 & & \\
\hline Nonstandard anticoagulation (no. [\%]) & $8(53.3)$ & $7(46.7)$ & 0.746 & & \\
\hline Midline shift >5 mm (no. [\%]) & $20(58.8)$ & $14(41.2)$ & 0.724 & & \\
\hline Mean lesion vol in ml (mean \pm SD) & $113.21 \pm 17.39$ & $116.60 \pm 18.36$ & 0.476 & & \\
\hline Lesion type (no. [\%]) & & & 0.001 & $6.857(2.146-21.907)$ & 0.247 \\
\hline Hemorrhage-dominated lesion & $9(33.3)$ & $18(66.7)$ & & & \\
\hline Edema-dominated lesion & $24(77.4)$ & $7(22.6)$ & & & \\
\hline Bilat lesions (no. [\%]) & $2(22.2)$ & $7(77.8)$ & 0.036 & $6.028(1.129-32.193)$ & 0.118 \\
\hline Multiple sinus involvement (no. [\%]) & $6(54.5)$ & $5(45.5)$ & 0.861 & & \\
\hline Deep cerebral venous involvement (no. [\%]) & $1(14.3)$ & $6(85.7)$ & 0.039 & 10.105 (1.129-90.454) & 0.136 \\
\hline
\end{tabular}

(46.6\%) as having hemorrhage-dominated lesion and the others (31 patients [53.4\%]) as having edema-dominated lesions. The superior sagittal sinus (26 cases [44.8\%]) was the most common site, and 7 patients suffered from deep CVT in combination with dural sinus involvement, the transverse sinus in 4 , the sigmoid sinus in 1 , and multiple sinuses in 2.

\section{Treatment and Complications}

Hematomas were associated with significant mass effect in 3 patients; therefore, hematoma evacuation was performed in these patients during DC. Intracranial pressure monitoring was used in 37 patients (63.8\%). The average daily postoperative ICP within the first 72 hours was $16.9 \pm 9.3 \mathrm{~mm} \mathrm{Hg}$ in patients with hemorrhage-dominated lesions and $12.7 \pm 8.2 \mathrm{~mm} \mathrm{Hg}$ in those with edema-dominated lesions. To maintain an optimal cerebral perfusion pressure (CPP; 60-70 mm Hg), 29 patients received second-tier ICP control via therapeutic hypothermia, which was continued for more than 48 hours in 16 patients. The mean lowest CPP increased from 56.4 to $63.6 \mathrm{~mm} \mathrm{Hg}$ after 24 hours of hypothermia.

Four patients underwent reoperation: 2 who underwent hematoma evacuation due to hemorrhage progression, 1 who received bone flap enlargement, and 1 who had contralateral DC. Subdural hygroma (14 cases [24.1\%]) was the most common postoperative complication, and all formed within 4 weeks after surgery. Four patients with subdural hygroma required contemporary subcutaneous drainage. One hygroma persisted for more than 6 months without causing symptoms or undergoing self-absorption; thus, a subdural peritoneal shunt procedure was per- formed. No cases of DC-related intracranial infection or hydrocephalus occurred.

\section{Follow-Up}

At the 6-month follow-up, 33 patients (56.9\%) had attained a favorable outcome (mRS score of 0 in 3 patients, score of 1 in 13, and score of 2 in 17), whereas 25 patients (43.1\%) had experienced a poor outcome (mRS score of 3 in 12 patients, score of 4 in 4 , score of 5 in 1 , and score of 6 in 8). Eight patients (13.8\%) died, including 6 who died from deterioration of the intracranial lesion, 1 who died of ventriculitis due to methicillin-resistant Staphylococcus aureus, which was attributed to external ventricular drainage, and 1 who died of multiple organ dysfunction syndrome. Of the patients with an mRS score of 4 or 5,3 exhibited significant cognitive deficits, 1 was in a persistent vegetative state, and 1 suffered from hemiplegia.

\section{Risk Factors}

After analyzing 14 preoperative candidate variables, we found age, duration from symptom onset to surgery, hemorrhage-dominated lesion, bilateral lesions, and deep cerebral venous involvement were considered potential risk factors for poor outcomes. (Because our sample size was so small and to reduce statistical bias and any chance of missing a potential risk factor, we considered all risk factors with a p value close to 0.05 in our analysis.) Table 2 summarizes the results of our univariate analysis. Results of the multivariate regression analysis revealed that hemorrhage-dominated lesions $(\mathrm{p}=0.026)$ and deep cerebral venous involvement $(p=0.026)$ were associated with poor 
TABLE 3. Multivariate logistic regression

\begin{tabular}{lcc}
\hline \multicolumn{1}{c}{ Variable } & $p$ Value & OR $(95 \% \mathrm{Cl})$ \\
\hline Age & 0.113 & $1.045(0.990-1.102)$ \\
\hline Duration from onset to op & 0.270 & $0.776(0.495-1.217)$ \\
\hline Hemorrhage-dominated lesion & 0.026 & $6.255(1.240-31.547)$ \\
\hline Bilat lesions & 0.182 & $4.177(0.512-34.053)$ \\
\hline Deep cerebral venous involvement & 0.026 & $16.254(1.396-189.321)$ \\
\hline
\end{tabular}

outcomes in patients with severe hemorrhagic CVT (Table $3)$. The results of the Hosmer-Lemeshow test $(p=0.313)$ indicated that the regression model was well calibrated. Poor outcomes could be explained by this model (Nagelkerke $\mathrm{R}^{2}$ ) in $51 \%$ of cases. The area under the curve was 0.839 .

\section{Discussion}

Cerebral venous thrombosis is an uncommon but potentially life-threatening stroke with unique features. ${ }^{18}$ Although the general outcome of CVT is favorable, ${ }^{11} \mathrm{se}-$ vere CVT can cause progressive intracranial hypertension resulting in brain herniation. ${ }^{3}$ For patients with impending herniation, DC may be the only life-saving treatment. However, the current guidelines do not elaborate on the benefits of and indications for DC in patients with CVT. Given the relatively low incidence ( 5 cases/1,000,000 persons) of this condition, ${ }^{2}$ only limited studies with small sample sizes have been reported (Table 4). 1,9,12-17,22,25 Therefore, more investigations regarding the correlations between clinicoradiological features and the surgical outcomes of severe CVT are expected, especially as regards severe hemorrhagic CVT.

\section{Incidence}

Because the inclusion criteria and surgical indications were diverse in previous studies, the incidence of DC for CVT varied from $1.4 \%(9 / 624)$ to $7.4 \%(44 / 587){ }^{1,8}$ According to the 3 largest series, patients with hemorrhagic CVT constituted $82 \%,{ }^{1} 89.9 \%,{ }^{9}$ and $100 \%{ }^{16}$ of all the CVT patients who underwent DC surgery. This finding may indicate that patients with hemorrhagic CVT are more likely to suffer from severe refractory intracranial hypertension. However, none of these studies reported the overall frequency of DC in patients with hemorrhagic CVT. In the present study, we focused on DC application in patients with hemorrhagic CVT. The surgical indications were a preoperative GCS score $\leq 9$ and progressive deterioration in neurological status despite the administration of maximal medical therapy. Consequently, the frequency of DC in patients with hemorrhagic CVT was $28.8 \%(58 / 201)$.

West China Hospital is the leading referral stroke center in western China, serving approximately 500 million people from more than 5 provinces. Tibetans constitute approximately $1.5 \%$ of the entire population in this area; however, they represented a much larger portion (13.8\%) of our sample. Tibetan individuals have long lived in highaltitude areas and consumed high-fat diets and thus face an increased risk of developing viscous hyperlipidemia. ${ }^{24}$
TABLE 4. Literature summary of studies on DC for CVT

\begin{tabular}{lcccc}
\hline \multicolumn{1}{c}{$\begin{array}{c}\text { Authors } \\
\& \text { Year }\end{array}$} & $\begin{array}{c}\text { No. of } \\
\text { Patients }\end{array}$ & $\begin{array}{r}\text { Favorable } \\
\text { Outcome } \\
\text { (no. [\%]) }\end{array}$ & $\begin{array}{r}\text { Poor } \\
\text { Outcome } \\
\text { (no. [\%]) }\end{array}$ & $\begin{array}{r}\text { Mortality } \\
\text { (no. [\%]) }\end{array}$ \\
\hline Keller et al., 2005 & 4 & $4(100.0)$ & $0(0.0)$ & $0(0.0)$ \\
\hline Théaudin et al., 2010 & 8 & $7(87.5)$ & $1(12.5)$ & $1(12.5)$ \\
\hline Lath et al., 2010 & 11 & $8(72.7)$ & $3(27.3)$ & $3(27.3)$ \\
\hline Mohindra et al., 2011 & 13 & $11(84.6)$ & $2(15.4)$ & $2(15.4)$ \\
\hline Ferro et al., 2011 & 69 & $39(56.5)$ & $30(43.5)$ & $11(15.9)$ \\
\hline Zuurbier et al., 2012 & 10 & $6(60.0)$ & $4(40.0)$ & $2(20.0)$ \\
\hline Rajan et al., 2012 & 34 & $26(76.5)$ & $8(23.5)$ & $6(17.6)$ \\
\hline Aaron et al., 2013 & 44 & $27(61.4)$ & $8(18.2)$ & $9(20.4)$ \\
\hline Raza et al., 2014 & 7 & $4(57.1)$ & $3(42.8)$ & $2(28.6)$ \\
\hline
\end{tabular}

We hypothesized that long-term residence in high-altitude areas and viscous hyperlipidemia may be responsible for the increased number of Tibetan patients who suffered from severe hemorrhagic CVT. But because of the inadequate evidence regarding blood hyperviscosity, the mechanism underlying the above relationship warrants further investigation.

\section{Outcomes}

Because of advances in neuroimaging and acute anticoagulation treatment, several studies have reported that CVT has a favorable prognosis with a mortality rate $<10 \%$ and that good functional outcomes are achieved in $>80 \%$ of patients. ${ }^{4,8}$ However, some patients can develop refractory intracranial hypertension and experience poor outcomes, especially patients with hemorrhagic lesions. ${ }^{4,7,10,23}$ The major hazard of severe CVT is herniation attributable to the mass effect produced by hemorrhagic venous infarctions. Different from large ischemic infarctions, the venous infarctions in CVT are caused by venous congestion. Decompressive craniectomy, as a life-saving treatment in affected patients, reduces the intracranial pressure and enhances the recirculation of cortical veins and collapsed veins. In addition, it reduces venous congestion, improves venous blood flow in the collateral circulation, and allows anticoagulants to reach the site of thrombosed veins. ${ }^{19,20}$ Thus far, however, only a limited number of studies with small samples have retrospectively analyzed the outcomes of patients with severe CVT who underwent DC (Table 4). ${ }^{1,9,12-17,22,25}$ Given the lack of universal surgical indications, the results of these studies vary with mortality rates ranging from $0 \%$ to $28.6 \%$ and good functional outcome rates ranging from $56.5 \%$ to $100 \%$. In the present study, the overall mortality rate was $13.8 \%$, and 33 patients $(56.9 \%)$ achieved good functional outcomes. Since the patients enrolled in our study were suffering from severe hemorrhagic CVT, their surgical outcomes were not as favorable as those in previous studies. Nevertheless, patients with severe hemorrhagic CVT who underwent DC experienced better outcomes than patients with severe traumatic brain injury with mass effect, spontaneous intracerebral hemorrhage, or large ischemic infarctions. 


\section{Risk Factors}

In previous studies, several factors such as male sex, age $>37$ years, acute symptom onset, seizures, coma, mental status disorder, GCS scores $<9$, hemorrhagic infarction, thrombosis of the deep cerebral venous system, posterior fossa involvement, central nervous system infection, and cancer were considered independent risk factors for poor outcomes in patients with CVT. ${ }^{3,8,10}$ However, because of the small sample sizes in these studies, the risk factors for more severe CVT, let alone those for severe hemorrhagic CVT, were difficult to identify. A registered multicenter study reported favorable outcomes in $82 \%$ of severe CVT patients who underwent DC and demonstrated that poor outcomes were more likely to occur in patients with coma and bilateral lesions. ${ }^{9}$ Aaron et al. found that performing DC within 12 hours after admission significantly increased patient survival rates, whereas surgery delayed by more than 12 hours had a significant effect on mortality. ${ }^{1}$ Admittedly, once the diagnosis of CVT has been confirmed, early surgery is appropriate for patients with impending herniation or established herniation. However, CVT patients may present with chronic progressive clinical courses, and venous infarctions generally have greater recovery potential than ischemic strokes. Given the good responses to medical treatment and the risks of DC-related complications, our institution performs DC in patients with impending or established herniation despite maximal medical therapy and the provision of intensive care. Additionally, in our study, 5 patients died during the acute stage (defined as the first 72 hours after symptom onset), whereas patients with a less severe condition experienced more gradual deterioration over several days and did not die. We concluded that the outcomes of severe hemorrhagic CVT were associated with patient clinical courses. Therefore, the duration between symptom onset and surgery was considered a potential risk factor for a poor outcome; however, given the small sample size of our study, the results did not show such a correlation.

In the present study, results of the multivariate regression analysis revealed that hemorrhage-dominated lesions and deep CVT were independent risk factors for poor outcomes in patients with severe hemorrhagic CVT. According to our institutional experience, in patients with severe hemorrhagic CVT, herniation is caused by mass effect secondary to both venous infarction and intracerebral hemorrhage. Hemorrhage may damage the surrounding brain tissue and cause worsening focal edema. Therefore, we hypothesized that the volume of intracerebral hemorrhage may be a risk factor for poor outcomes in patients with severe hemorrhagic CVT. However, since hemorrhage in CVT appears as a scattered hyperdense lesion on $\mathrm{CT}$, accurate measurements of hemorrhage volume are not possible. Therefore, we introduced the $\mathrm{H} / \mathrm{L}$ ratio as a simple measurement that allowed us to divide the severe hemorrhagic CVT into the following 2 groups: 1) Hemorrhage-dominated lesions were characterized by an $\mathrm{H} / \mathrm{L}$ ratio $>0.5$, which represented a major hemorrhage surrounded by moderate edema on neuroimaging; and 2) edema-dominated lesions were characterized by an $\mathrm{H} / \mathrm{L}$ ratio $<0.5$, which represented a large area of edema mixed with small, scattered, patchy hemorrhages. In the present study, we found that hemorrhage-dominated lesions were associated with poorer outcomes. Additionally, we did not observe a significant correlation between lesion volumes and patient outcomes, which further proved that volume of hemorrhage played an important role in clinical outcome in patients with severe hemorrhagic CVT.

Deep cerebral venous involvement was considered an independent risk factor for a poor outcome in our study. Patients with deep CVT were at risk for developing an obstruction in the vein of Galen, which can result in damage to the thalamus and basal ganglia. Results of the multivariate regression analysis showed that patients who suffered from severe hemorrhagic CVT with deep venous involvement were 2.3 times more susceptible to poor outcomes than the patients without deep venous involvement.

\section{Study Limitations}

This was an institutional long-term retrospective study of patients with severe hemorrhagic CVT with impending or established herniation. The sample size was relatively limited, which may have resulted in statistical bias. Because of ethical concerns, it was not possible to include a control group. Additionally, because of financial limitations and local medical insurance policy restrictions, we could not obtain sufficient data regarding genetic or acquired prothrombotic conditions. Therefore, we did not perform a risk analysis toward laboratory variables, but we are aware that these variables may play an important role in the progression and recurrence of this disease. Moreover, the time span was quite long, and some of the patients enrolled in the study were from remote villages in Tibet; therefore, some were lost to follow-up since they were unable to travel a long distance for their return visit. This made long-term follow-up problematic; thus, short-term follow-up was adopted in our study.

\section{Conclusions}

In patients with severe hemorrhagic CVT, DC is considered an effective life-saving treatment that can result in favorable outcomes. Notably, our study showed that hemorrhage-dominated lesions and deep cerebral venous involvement were independent risk factors for a poor outcome in patients with this disease.

\section{Acknowledgments}

This study was funded by the National Science \& Technology Pillar Program during the 12th Five-Year Plan Period (No. 2011BAI08B05).

\section{References}

1. Aaron S, Alexander M, Moorthy RK, Mani S, Mathew V, Patil AK, et al: Decompressive craniectomy in cerebral venous thrombosis: a single centre experience. J Neurol Neurosurg Psychiatry 84:995-1000, 2013

2. Bousser MG, Ferro JM: Cerebral venous thrombosis: an update. Lancet Neurol 6:162-170, 2007

3. Canhão P, Ferro JM, Lindgren AG, Bousser MG, Stam J, Barinagarrementeria F: Causes and predictors of death in cerebral venous thrombosis. Stroke 36:1720-1725, 2005

4. Dentali F, Gianni M, Crowther MA, Ageno W: Natural his- 
tory of cerebral vein thrombosis: a systematic review. Blood 108:1129-1134, 2006

5. deVeber G, Andrew M, Adams C, Bjornson B, Booth F, Buckley DJ, et al: Cerebral sinovenous thrombosis in children. N Engl J Med 345:417-423, 2001

6. Einhäupl K, Stam J, Bousser MG, De Bruijn SF, Ferro JM, Martinelli I, et al: EFNS guideline on the treatment of cerebral venous and sinus thrombosis in adult patients. Eur J Neurol 17:1229-1235, 2010

7. Ferro JM, Canhão P, Bousser MG, Stam J, Barinagarrementeria F, Stolz E: Cerebral venous thrombosis with nonhemorrhagic lesions: clinical correlates and prognosis. Cerebrovasc Dis 29:440-445, 2010

8. Ferro JM, Canhão P, Stam J, Bousser MG, Barinagarrementeria F: Prognosis of cerebral vein and dural sinus thrombosis: results of the International Study on Cerebral Vein and Dural Sinus Thrombosis (ISCVT). Stroke 35:664-670, 2004

9. Ferro JM, Crassard I, Coutinho JM, Canhão P, Barinagarrementeria F, Cucchiara B, et al: Decompressive surgery in cerebrovenous thrombosis: a multicenter registry and a systematic review of individual patient data. Stroke 42:28252831, 2011

10. Girot M, Ferro JM, Canhão P, Stam J, Bousser MG, Barinagarrementeria $\mathrm{F}$, et al: Predictors of outcome in patients with cerebral venous thrombosis and intracerebral hemorrhage. Stroke 38:337-342, 2007

11. Hiltunen S, Putaala J, Haapaniemi E, Tatlisumak T: Longterm outcome after cerebral venous thrombosis: analysis of functional and vocational outcome, residual symptoms, and adverse events in 161 patients. J Neurol 263:477-484, 2016

12. Keller E, Pangalu A, Fandino J, Könü D, Yonekawa Y: Decompressive craniectomy in severe cerebral venous and dural sinus thrombosis. Acta Neurochir Suppl 94:177-183, 2005

13. Lanterna LA, Gritti P, Manara O, Grimod G, Bortolotti G, Biroli F: Decompressive surgery in malignant dural sinus thrombosis: report of 3 cases and review of the literature. Neurosurg Focus 26(6):E5, 2009

14. Lath R, Kumar S, Reddy R, Boola GR, Ray A, Prabhakar S, et al: Decompressive surgery for severe cerebral venous sinus thrombosis. Neurol India 58:392-397, 2010

15. Mohindra S, Umredkar A, Singla N, Bal A, Gupta SK: Decompressive craniectomy for malignant cerebral oedema of cortical venous thrombosis: an analysis of 13 patients. Br J Neurosurg 25:422-429, 2011

16. Rajan Vivakaran TT, Srinivas D, Kulkarni GB, Somanna S: The role of decompressive craniectomy in cerebral venous sinus thrombosis. J Neurosurg 117:738-744, 2012

17. Raza E, Shamim MS, Wadiwala MF, Ahmed B, Kamal AK: Decompressive surgery for malignant cerebral venous sinus thrombosis: a retrospective case series from Pakistan and comparative literature review. J Stroke Cerebrovasc Dis 23:e13-e22, 2014
18. Saposnik G, Barinagarrementeria F, Brown RD Jr, Bushnell CD, Cucchiara B, Cushman M, et al: Diagnosis and management of cerebral venous thrombosis: a statement for healthcare professionals from the American Heart Association/ American Stroke Association. Stroke 42:1158-1192, 2011

19. Soyer B, Rusca M, Lukaszewicz AC, Crassard I, Guichard JP, Bresson D, et al: Outcome of a cohort of severe cerebral venous thrombosis in intensive care. Ann Intensive Care 6:29, 2016

20. Stam J: Thrombosis of the cerebral veins and sinuses. N Engl J Med 352:1791-1798, 2005

21. Stam J, Majoie CB, van Delden OM, van Lienden KP, Reekers JA: Endovascular thrombectomy and thrombolysis for severe cerebral sinus thrombosis: a prospective study. Stroke 39:1487-1490, 2008

22. Théaudin M, Crassard I, Bresson D, Saliou G, Favrole P, Vahedi K, et al: Should decompressive surgery be performed in malignant cerebral venous thrombosis?: a series of 12 patients. Stroke 41:727-731, 2010

23. Wasay M, Bakshi R, Bobustuc G, Kojan S, Sheikh Z, Dai A, et al: Cerebral venous thrombosis: analysis of a multicenter cohort from the United States. J Stroke Cerebrovasc Dis 17:49-54, 2008

24. Zhao Y, Yao Z, D'Souza W, Zhu C, Chun H, Zhuoga C, et al: An epidemiological survey of stroke in Lhasa, Tibet, China. Stroke 41:2739-2743, 2010

25. Zuurbier SM, Coutinho JM, Majoie CB, Coert BA, van den Munckhof P, Stam J: Decompressive hemicraniectomy in severe cerebral venous thrombosis: a prospective case series. J Neurol 259:1099-1105, 2012

\section{Disclosures}

The authors report no conflict of interest concerning the materials or methods used in this study or the findings specified in this paper.

\section{Author Contributions}

Conception and design: Li. Acquisition of data: Zhang. Analysis and interpretation of data: Zhao. Drafting the article: Zhang. Critically revising the article: Hui. Reviewed submitted version of manuscript: Hui. Statistical analysis: Zhang. Study supervision: You.

\section{Correspondence}

Hao Li, Department of Neurosurgery, West China Hospital of Sichuan University, 37 Guo Xue Xiang, Wu Hou District, Chengdu 610041, People's Republic of China. email: lihao7951@ sina.com. 